\title{
Determination of the building products reliability parameters at different loads in a two- step mode
}

\author{
Nadezhda Chiganova* \\ Moscow State University of Civil Engineering, Yaroslavskoe shosse, 26, Moscow, 129337, Russia
}

\begin{abstract}
The reliability determination of building structures and their components in a two-step mode nowadays is a relevant task. The article studies the problem of determining the building structures and their components reliability performance in a two-step mode based on the results of the tests described. These tests were conducted as follows: the batch of products intended for test are first tested in the first mode, and then in the second mode. A certain moment of time for mode switching is selected randomly by a specified law. The product is then tested in the variable two-stage mode. During testing, moments of time of product operation in the first and second modes are registered. Two experimentation programs that differ in the way of mode switching are proposed: program tests and dynamic tests. Examples of the methodology application to the determination of the product failure rate were discussed. According to the results of theoretical and practical application of the proposed method, the way to determine the reliability parameters using different laws was suggested for application in structural analysis and construction products reliability assessment process.
\end{abstract}

\section{Introduction}

The solution to many of the reliability theory problems $[1,3,4,5]$ requires building structures and their components (hereinafter - "products") testing in the two-stage mode. Such problems include, for example, stress test problems, reliability calculation in the variable mode, life utilization principles validation of the products, replacement of the operation modes with laboratory ones. During structural calculation process, it is often necessary to evaluate the effect of certain parameters, which are random variables. Their laws of distribution are shown in table $1[8,9,10]$.

\footnotetext{
* Corresponding author: chiganovanm.mgsu@gmail.com
} 
Table 1. The laws of the distribution of random variables encountered during structural calculation process

\begin{tabular}{|l|l|}
\hline \multicolumn{1}{|c|}{ Random variables } & \multicolumn{1}{|c|}{ Type and name of the distribution law } \\
\hline Wind load & $\begin{array}{l}f(x)=\frac{1}{\sigma \sqrt{2 \pi}} \exp \left[-(x-a)^{2} / 2 \sigma^{2}\right],-\infty<x<\infty \\
\text { Normal }\end{array}$ \\
\hline Snow load & $\begin{array}{l}\text { a) } f(x)=\frac{1}{\sigma \sqrt{2 \pi}} \exp \left[-(\log x-a)^{2} / 2 \sigma^{2}\right], x>0 \\
\text { Lognormal } \\
\text { b) } \mathrm{f}(x)=\frac{x^{n_{1}}}{n_{1} !} \exp (-x) \\
x \geq 0, n_{1}=0,1,2 \ldots \\
\text { Poisson }\end{array}$ \\
\hline All loads & $\begin{array}{l}\text { a) } f(r)=\frac{r}{D_{x}} \exp \left(-r^{2} / 2 D_{x}\right), 0<r<\infty \\
\text { Rayleigh } \\
\text { b) } f(x)=\exp \left[-C_{1} \exp \left(-\alpha_{1} x\right)\right] \\
C_{1}>0, \alpha_{1}>0,-\infty<x<\infty\end{array}$ \\
\hline Durability parameters of the material $-\infty$ & a) $f(x)=\left\{\frac{\eta}{\sigma}\left(\frac{x}{\sigma}\right)^{\eta-1} \exp \left[-\left(\frac{x}{\sigma}\right)^{\eta}\right], x \geq 0\right.$ \\
0, in other cases & \\
& Weibull
\end{tabular}

Tests in two-step regime are as follows. The batch of products intended for test are first tested in the mode $\varepsilon_{1}$, and then in the mode $\varepsilon_{2}$. Moment $\tau$ of time for mode switching is selected randomly by a specified law $\mathrm{H}(\mathrm{t})=\mathrm{P}(\mathrm{t}<\tau)$. The product is tested in the variable twostage mode: $\varepsilon=\left\{\varepsilon_{1}, 0 \leq \mathrm{t}<\tau ; \varepsilon_{2}, \tau \leq \mathrm{t}<\infty\right\}$, where $\tau$ - random variable distributed according to the law $\mathrm{H}(\mathrm{t})$.

The article studies the problem of determining the building structures and their components reliability performance in mode $\varepsilon_{1}$ and $\varepsilon_{2}$ based on the results of the tests described. Let us set $\xi_{\mathrm{i}}$ and $\tilde{\xi}$ product failure time in the modes $\varepsilon_{i}$ and $\tilde{\varepsilon}$, respectively and through $F_{i}$ and $\tilde{F}$ their distribution function, i.e. $F_{i}(t)=P\left(\xi_{i}<t\right)$, i=1.2, $\tilde{F}(t)=P(\tilde{\xi}<t)$.

\section{Determination of the building structures and their components reliability performance}

Note [2] that there is the following relationship between random variables $\theta_{1}, \theta_{2}$ and $\xi_{\mathrm{i}}, \tilde{\xi}$ : $\theta_{1}=\min \left\{\xi_{i}, \tau\right\}, \theta_{2}=\max \{0, \tilde{\xi}-\tau\}$.

As the characteristics $\theta_{1}, \theta_{2}$ and $\tilde{\xi}$ are observed during the test, after the experimental test distribution functions $\tilde{F}(t), \theta_{i}(t)=P\left(\theta_{i}<t\right), i=1.2$ and $S(t)=P\left(\theta_{2}>t / \xi_{i}>\tau\right)$ can be restored. $\mathrm{S}(\mathrm{t})$ function is the conditional probability that the product not failed in the first mode during its testing on the program $\widetilde{\varepsilon}$, will operate in the second mode for no longer than t. Subsequently we distinguish between two experimentation programs [6,7] that differ in the way of mode switching.

A) Program tests. They consist in the fact that before the experiments start they are specified by the distribution function $H(t)$. Further, $n$ random values $\mu_{1}, \mu_{2}, \ldots, \mu_{n}$, evenly distributed by the law are determined by the random number tables corresponding to the number $\mathrm{n}$ of test products. Values $\tau_{i}=H^{-1}\left(\mu_{i}\right), i=1,2, \ldots, n$ are calculated, where $\mathrm{H}^{-1}-$ 
inverse $\mathrm{H}$ function. Product number $i$ is tested first in the mode $\varepsilon_{1}$ for a time $\tau$, then, if it does not fail - in the mode $\varepsilon_{2}$. Any function $H$ that has the probability density $h(t)>0$ at all t> 0 can be chosen.

B) Dynamic tests. In these tests, the mode switching is carried out only in the product failure times. Briefly the test sequence will be as follows. At first, all products are tested in the mode $\varepsilon_{1}$. Let's assume that the first failure occurred in the time t. Then randomly selected not failed product is switched to the second mode, and the remaining $n-2$ continue to be tested in the mode $\varepsilon_{1}$ as long as one of them fails. Assume that it happens in the moment of time $\tau_{2}$. Again, randomly selected not failed product is switched to the second mode, and the remaining n-4 continue to be tested in the first mode, etc. It is evident that in this way each product will be tested in the two-step mode of (I) type.

So, we will proceed from the fact that similar tests were carried out and, based on their results, distributions $\mathrm{Q}_{\mathrm{i}}(\mathrm{t}), \mathrm{S}(\mathrm{t})=\mathrm{P}(\mathrm{t})\left(\mathrm{Q}_{2}>\mathrm{t}, \tau_{1}>\imath\right)$ were obtained. First let us set a goal to find the distribution of products failure-free operation in the first mode $\bar{F}_{1}(t)=1-F_{1}(t)$ by received $\mathrm{Q}_{\mathrm{i}}, \mathrm{S}$.

1. Let the experiment be carried out under program A) and based on its results function $\mathrm{H}, \mathrm{Q}_{\mathrm{i}}, \mathrm{S}$ are obtained. It follows from the definition of random variable $\theta_{1}$ that

$$
\begin{aligned}
\theta_{1}(\mathrm{e})=P\left(\theta_{1}<t\right) & =P\left(\min \left\{\xi_{1}, \tau\right\}<t\right)=1-P\left(\xi_{1}>t, \tau<t\right) \\
= & 1-\left[1-F_{1}(t)\right][1-H(t)]=1-\bar{F}_{1}(t) \bar{H}(t), \bar{Q}_{1}(t)=\bar{F}_{1}(t) \bar{H}(t)
\end{aligned}
$$

From formula (2) we obtain the reliability function in the first mode

$\bar{F}_{1}(t)=\frac{\bar{Q}_{1}(t)}{\bar{H}(t)}, \bar{F}_{1}(t)=1-F_{1}(t)=P\left(\xi_{1}>t\right)$

We shall write the numerical characteristics of this distribution: mathematical expectation and variance of random variable:

$M(\xi)=\int_{0}^{\infty} \bar{F}_{1}(t) d t=\int_{0}^{\infty} \frac{\bar{Q}_{1}(t)}{\bar{H}(t)} d t, D\left(\xi_{1}\right)=M\left(\xi_{1}^{2}\right)-M^{2}(\xi)$

It should be noted that

$M\left(\xi_{1}^{2}\right)=2 \int_{0}^{\infty} t \bar{F}_{1}(t) d t=2 \int_{0}^{\infty} \frac{\bar{Q}_{1}(t)}{\bar{H}(t)} d t$

2. Let the tests be carried out under the programs B). In this case the switching distribution $\mathrm{H}(\mathrm{t})$ will not be known because it coincides with $F_{1}(t)$. Applying $H=F_{l}$ in (2), we obtain

$F_{1}(t)=\sqrt{\bar{Q}(t)}$

Formulas (2) and (3) show that in order to determine the product reliability characteristics in the first mode it is sufficient to find the lifetime distribution $Q_{l}$ based on the test conducted results $\theta_{1}$.

Although formulas (2) and (3) are simple, their use in practice may give rise to some difficulties. In fact, after the experimental study failure time will be known, and it is difficult to determine the distribution type by this data.

Therefore, $\bar{Q}_{1}$ functions for a given failures distribution of $F_{1}$ and $H$ are a matter of interest. 


\section{Application examples}

1. Let the products fail in the first mode exponentially $F_{1}(t)=1-e^{-\lambda_{1} t}$ and switching points are also set exponentially $H(t)=1-\exp \left(-\lambda_{2} t\right)$. Then $\bar{Q}_{1}(t)=\bar{F}_{1}(t) H(t)=\exp \left[-\left(\lambda_{1}+\lambda_{2}\right) t\right]$.

$M\left(\theta_{1}\right)=\int_{0}^{\infty} \bar{Q}_{1}(t) d t=\int_{0}^{\infty} \exp \left[-\left(\lambda_{1}+\lambda_{2}\right) t\right] d t=\frac{1}{\lambda_{1}+\lambda_{2}}$

We deduce from the last equation the failure rate in the first mode:

$\lambda_{1}=\frac{1-\lambda_{2} M\left(\theta_{1}\right)}{M\left(\theta_{1}\right)}$

2. Failures time is distributed by Poisson law: $F_{1}(t)=t^{n} e^{-t} / n$ ! and switching times are chosen exponentially: $H(t)=1-\exp (-\lambda t)$. In this case:

$$
\begin{aligned}
& \bar{Q}_{1}(t)=e^{-\lambda t}\left(1-\frac{t^{n} e^{-t}}{n !}\right), M\left(\theta_{1}\right)=\int_{0}^{\infty}\left[e^{-\lambda t}-\frac{t^{n} e^{-t(1+\lambda)}}{n !}\right] d t=\frac{1}{\lambda}-\frac{1}{(1+\lambda)^{n+1}}, \text { where } \\
& (1+\lambda)^{n+1}=\frac{\lambda}{1-\lambda M\left(\theta_{1}\right)}, n=\log _{(\lambda+1)}\left[\frac{\lambda}{1-\lambda M\left(\theta_{1}\right)}\right]-1 .
\end{aligned}
$$

\section{Determination of the reliability parameters using different laws}

Table 2 presents the results of calculating the function $\bar{\theta}_{1}(t)$, representing the failure function calculated by the formula (1) with different laws of failure $F_{1}(t)$. Functions $F_{1}(t)$ were selected from table 1 depending on the nature of the load. For example, if the failure of the test product occurred due to wind load, then the law of failure $F_{1}(t)$ was chosen as normal. If the snow load is considered, the failure of the product in the first mode is selected according to the Poisson law, etc.

The moment of switching from the first to the second mode was considered as exponential $H(t)=1-\exp \left(-\lambda_{2} t\right)$. Then a numerical characteristic, such as the mathematical expectation $M\left(\theta_{1}\right)$ and $M\left(\theta_{2}\right)$ for random variables was considered $\theta_{1}$ and $\theta_{2}$ - time of work in the first and second modes, characterizing the average time of work in each mode. The following functions are used in the table:

1. $\Phi(x)$ - normal distribution function

$\Phi(x)=\frac{1}{\sqrt{2 \pi}} \int_{-\infty}^{x} \bar{e}^{t^{2} / 2} d t$

2. $\Gamma(\alpha)$ - Gamma function

$$
\Gamma(\alpha)=\int_{0}^{\infty} t^{\alpha-1} e^{-t} d t(\operatorname{Re} \alpha>0)
$$

3. $\gamma(\alpha, x)$-incomplete gamma function

$$
\gamma(\alpha, x)=\int_{0}^{x} t^{\alpha-1} e^{-t} d t
$$

Table 2 Numerical Characteristics for the Exponential Law of Switching Modes H(t)

\begin{tabular}{|l|l|}
\hline \multicolumn{1}{|c|}{ Law of Failure } & \multicolumn{1}{c|}{ Numerical Characteristics } \\
\hline Exponential & $\overline{Q_{1}(t)}=\exp \left[-\left(\lambda_{1}+\lambda_{2}\right) t\right] ;$ \\
$F_{1}(t)=1-e^{-\lambda_{1} t}, \mathrm{t} \geq 0, \lambda_{1} \geq 0$ & $M\left(\theta_{1}\right)=\frac{1}{\lambda_{1}+\lambda_{2}}$ \\
\hline
\end{tabular}




\begin{tabular}{|c|c|}
\hline $\begin{array}{l}\text { Gamma distribution } \\
f_{1}(t)=\frac{\lambda_{1}^{\alpha_{1}} t^{\alpha_{1}-1}}{\Gamma\left(\alpha_{1}\right)} \exp \left(-\lambda_{1} t\right) \\
F_{1}(t)=\frac{\gamma\left(\alpha_{1}, \lambda_{1}, t\right)}{\Gamma\left(\alpha_{1}\right)} \\
\alpha_{1}>0, \quad \lambda_{1}>0, \quad t \geq 0\end{array}$ & $\begin{array}{l}Q_{1}(t)=\left[1-\frac{\gamma\left(\frac{n_{1}}{2} ; \frac{t}{2}\right)}{\Gamma\left(\frac{n_{1}}{2}\right)}\right] \exp \left(-\lambda_{2} t\right) \\
M\left(\theta_{1}\right)=1 / \lambda_{2}-\left(1+\lambda_{2}\right)^{-\alpha_{1}} / \lambda_{1} \lambda_{2} \\
M\left(\theta_{2}\right)=\frac{2}{\lambda_{2}^{2}}- \\
-\frac{2 \lambda_{1}^{\alpha_{1}} \Gamma\left(2+\alpha_{1}\right)_{2} F_{1}\left(1,2+\alpha_{1} \alpha_{1}+1 \frac{\lambda_{1}}{\lambda_{1}+\lambda_{2}}\right)}{\alpha_{1}\left(\lambda_{1}+\lambda_{2}\right)^{2+\alpha_{1} \Gamma\left(\alpha_{1}\right)}}\end{array}$ \\
\hline $\begin{array}{l}\chi^{2} \text { - distribution } \\
f_{1}(t)=\frac{t^{\left(\frac{n_{1}}{2}-1\right)} \exp \left(-\frac{t}{2}\right)}{2^{\frac{n_{1}}{2}} \Gamma\left(\frac{n_{1}}{2}\right)} \\
F_{1}(t)=\frac{\gamma\left(\frac{n_{1}}{2}, \frac{t}{2}\right)}{\Gamma\left(\frac{n_{1}}{2}\right)} \\
t \geq 0, \quad n_{1}=0,1,2 \ldots\end{array}$ & $\begin{array}{l}\bar{Q}_{1}(t)=\left[1-\frac{\gamma\left(\frac{n_{1}}{2} ; \frac{t}{2}\right)}{\Gamma\left(\frac{n_{1}}{2}\right)}\right] \exp \left(-\lambda_{2} t\right) ; \\
M\left(\theta_{1}\right)=\frac{1}{\lambda_{2}^{2}}-\frac{2}{\lambda_{2}}\left(1+\lambda_{2}\right)^{-\frac{n_{1}}{2}}\end{array}$ \\
\hline $\begin{array}{l}\text { Normal } \\
f_{1}(t)=\frac{1}{\sigma_{1} \sqrt{2 \pi}} \exp \left[-\frac{\left(t-a_{1}\right)^{2}}{2 \sigma_{1}^{2}}\right. \\
F_{1}(t)=\Phi\left(\frac{t-a_{1}}{\sigma_{1}}\right) \\
-\infty<t<\infty, \sigma_{1}>0\end{array}$ & $\begin{array}{l}\overline{Q_{1}}(t)=\Phi\left(\frac{t-a_{1}}{\sigma_{1}}\right) \exp \left(-\lambda_{2} t\right) \\
M\left(\theta_{1}\right)=\frac{1}{\lambda_{2}}-\frac{1}{x_{2}} \exp \left(\frac{\sigma_{1}^{2} \lambda_{2}^{2}}{2}-\lambda_{2} a_{1}\right) \times \\
\times\left[1-\Phi\left(\lambda_{2} \sigma_{1}\right)\right] ; \\
M\left(\theta_{2}^{2}\right)=\frac{1}{\lambda_{2}^{2}}-\frac{2}{\lambda_{2}} \exp \left(\lambda_{2}^{2} \sigma_{1}^{2}-\lambda_{2} a_{1}\right) \times \\
\times\left\{\left[1-\Phi\left(\lambda_{1} \sigma_{2}\right)\right]\left(\frac{1}{\lambda_{2}}-\lambda_{2} \sigma_{1}^{2}+a_{1}\right)+\frac{\sigma_{1}}{\sqrt{2 \pi}} \exp -\left(\frac{\sigma_{1}^{2} \lambda_{2}^{2}}{2}\right.\right.\end{array}$ \\
\hline $\begin{array}{l}\text { Double exponential } \\
F_{1}(t)=\exp \left[-C_{1} \exp \left(-\alpha_{1} t\right)\right] \\
C_{1}>0, \quad \alpha_{1}>0,-\infty<t<\infty\end{array}$ & $\begin{array}{l}Q(t)= \\
=\left\{1-\exp \left[-C_{1} \exp \left(-a_{1} t\right)\right]\right\} \exp \left(-\lambda_{2} t\right) \\
M\left(\theta_{1}\right)=1 / \lambda_{2}-C_{1}^{-\lambda_{2} / \lambda_{1}} \gamma\left(\lambda_{2} / \lambda_{1}, C_{1}\right) / a_{1} ; \\
M\left(\theta_{2}^{2}\right)=\frac{2}{\lambda_{2}^{2}}-2 C_{1}^{-\lambda_{2} / \lambda_{1}} \times \\
\times\left[\gamma\left(\lambda_{2} / \lambda_{1}, C_{1}\right)-\left(\frac{\lambda_{2}}{\lambda_{1}}-1\right) \gamma\left(\lambda_{2} / \lambda_{1}-1, C_{1}\right)\right] / \alpha_{1}^{2}\end{array}$ \\
\hline $\begin{array}{l}\text { Poisson } \\
F_{1}(t)=\frac{t^{n_{1}}}{n_{1} !} \exp (-t) \\
t \geq 0, n_{1}=0,1,2 \ldots\end{array}$ & $\begin{array}{l}\overline{Q_{1}}(t)=\left[1-\frac{t^{n_{1}}}{n_{1} !} \exp (-t)\right] \exp \left(-\lambda_{2} t\right) \\
M\left(\theta_{1}\right)=1 / \lambda_{2}-1 /\left(1+\lambda_{2}\right)^{n_{1}+1}\end{array}$ \\
\hline
\end{tabular}

\section{Conclusions}

The results obtained in the framework of the study on changes in reliability indicators in a two-step mode allow us to simplify the assessment of the reliability of constriuction products when changing test modes. In addition, using the data presented in Table 2, one can immediately determine the numerical characteristics with different laws of failure distribution in the first mode of the tested products in two-stage mode, which can be encountered in the structural analysis and construction products reliability assessment process. Further application and development of the proposed method may allow to produce complex optimization of the currently existing methods for reliability assessment using various automated systems. 


\section{References}

1. B.V. Gnedenko, J.K. Beljaev, A.D. Solov'ev, Mathematical methods in reliability theory (Nauka, 2010)

2. N.M. Chiganova, Natural and Technical Sciences 6, 14-17 (2015)

3. V. Medvedev, A. Pustovgar, Applied Mechanics and Materials 725-726, 337-382 (2015)

4. G.D. Kartashov, N.M. Chiganova, Journal of Mathematical Sciences 39, 2578-2588 (1987)

5. G.D. Kartashov, Elements and schemes accelerated testing (monograph) (Knowledge, 1990)

6. I.A. Ushakov, Reliability: Theory \& Applications 2, 6-19 (2007)

7. G.D. Kartashov, Theory of Probability and its Applications 14(4), 595-611 (1969)

8. V.A. Lomakin, Statistical problems of mechanics of rigid and deformable bodies 2, 114 (2004)

9. Z.I. Blokhovyak Analysis of the choice of the optimal method in construction with changeable parameters 1, 211 (2015)

10. Recommendations for assessing the reliability of building structures (Guidelines) (UralprostroyNIIproekt, 2001) 